\title{
Analysis of sediment abrasion potential in hydro turbines by studying fine sediments from the Budhi Gandaki-Trishuli River of Nepal
}

\author{
Roman Gantawa and *Naresh Kazi Tamrakar \\ Central Department of Geology, Tribhuvan University, Kirtipur, Kathmandu, Nepal \\ *Corresponding author: ntamrakar@hotmail.com
}

\begin{abstract}
Hydropower projects is considered to be one of the most cost-effective, environment friendly technologies for power generation. In hydropower plants, turbine is an important component and it has many associate problems that degrade its condition and cause reduction in efficiency, increase operational and maintenance costs. This research was adopted to analyse properties of sediment that are responsible for erosion in turbine. The study was focused on sediment properties; grain size, shape, mineral composition, hardness index and abrasivity. Two grain size fractions, i.e. 250 microns to pan fraction and 500-250 micron-sands were studied. It was depicted that the smaller size-fractions of the Budhi Gandaki-Trishuli River sediment contained more angular and elongate grains that the large size-fractions. Sediments contained more than $50 \%$ quartz, $8-17 \%$ feldspar, $10-20 \%$ mica, $5-10 \%$ fine lithic fragments, $5-12 \%$ coarse lithic fragments, and less than $1 \%$ carbonate lithic fragments, and heavies. In both smaller and larger size fractions, S5 samples (Rampur tar) possessed the highest abrasion rate of respectively $7.57 \mathrm{mg} / \mathrm{g}$ per hour and $13.75 \mathrm{mg} / \mathrm{g}$ per hour in test specimen. Abrasion rate tends to increase with reduction in roundness value and increase in proportion of quartz in sediments. The relationship between abrasivity and mean roundness is more well explained by the coarser size-fraction (500-250 microns) than the finer size-fraction, whereas abrasivity vs. \%quartz is better explained by the finer size-fractions than the coarser ones.
\end{abstract}

Keywords: Mineral composition, Sediment abrasion, Budhi Gandaki, Hydro turbine, Rotating disc apparatus

Paper Received: 31 Dec 2018

Paper Accepted: 6 Apr 2019

\section{INTRODUCTION}

Nepal has more than six thousand small and large streams, snowcapped mountains and glaciers in the Himalayas. The perennial rivers in steep gradient flow from high elevation to low land regions providing suitable conditions for the development of hydroelectric projects in Nepal. Shrestha (1966) revealed that hydropower development capacity of Nepal is $83,000 \mathrm{MW}$ of which $43,000 \mathrm{MW}$ is economically feasible. Parajuli (2003) also roughly estimated total hydropower potential 1,26,000 MW including smaller catchments. But only 1016 MW electricity have been generating at present, according to Department of Electricity Development on March 2019.

In Nepal, most of the hydropower plants are being constructed in the run-off-river system (ROR) except for Kulekhani HEP, and all projects have been affected to some extent by sand erosion. ROR projects contribute more than $80 \%$ of the total hydropower production in Nepal and the biggest hydropower project Kali Gandaki-A (144 MW) in Nepal is a ROR type. The Francis turbines of Panauti, Trishuli and Sunkoshi were eroded frequently and restored by welding and grinding. Even with well-designed sediment settling and flushing systems, power plants like Marsyangdi, Khimti and Jhimruk are having severe erosion problems (Thapa, 2004; Thapa et al., 2005). The turbine at Jhimruk Power Plant (12 MW capacity) in Nepal is an example of how the sediment erosion effects the power plant operation. The power plant has been been facing considerable sediment erosion as a result, it needs to be repaired on an annual basis (Pradhan, 2004). The turbines need to be maintained annually due to high erosion wear. The quartz content in Jhimruk is highest among the other rivers (Basnyat, 1999).

Study of abrasion in hydro turbine components is complex which is governed by various parameters; (1) Characteristics of eroding particles: their size, shape and hardness, mineral constituent proportion, and toughness which have a direct impact on turbine material, (2) Turbine base material, a coating of turbine and (3) Operating conditions: velocity, impingement angle and concentration (Mann, 2000; Thapa, 2004a). Sediment erosion is a phenomenon of mechanical wear of components. According to the Kjolle (2003) damages concerning water turbines are caused mainly by cavitation problems, sand erosion and material defects. This is due to the dynamic action of sediment flowing along with water impacting against a solid surface of hydraulic components. Therefore, sediment flowing along with water passing through the turbine is the root cause of sediment erosion in turbine components. The mechanical wear in hydraulic machinery is mainly due to the suspended sediment in the water, which is subjected to kinetic energy, the force of gravity, viscosity, turbulence, centrifuge and cavitation. Sand erosion is prominent for resulting a decrease in turbine efficiency. Sediment load is one of the major factors for wear and tear of turbines (Basnyat, 1997; Bajracharya et al., 2008). Even one percent loss of turbine efficiency leads to a remarkable 
decrease in power generation along with the high economic loss. Nepalese hydropower plants are more vulnerable to sand erosion as most of the rivers are originated from the Himalaya and contain high sediment concentrations along with a high percentage of quartz in the sediment. The very small sand particle that is not trapped in the settling basin passes to the turbine and causes abrasion. Typical cutting action, grooving and scooping of by abrasive quartz particles causes removal of turbine material.

Both storage as well as Run-of-River schemes of hydropower projects suffer significantly from sediment erosion problems but the nature of problem is different. The Run-ofRiver projects often suffer from erosion of turbines. Thus, nature of sediments in the river should be carefully analysed during feasibility design stage of hydropower projects as it results in loss of efficiency, reliability, revenue and cost, etc. Therefore, the main objectives of present study were (i) to assess the composition and texture of the river sediment and point out sites where sediment have low or high abrasivity between the Main Central Thrust (MCT) and the Main Boundary Thrust (MBT), and (ii) to find how does abrasion potential vary with respect to sediment size, texture, mineral content and the downstream sites of the river.

\section{METHODS}

Representative samples were collected along bar deposit of the Budhi Gandaki River segment upto the segment of the Trishuli River within the MCT and the MBT (Fig. 1). They were then sieved in the field, and size passing from 500-micron were collected to bring into the laboratory. Mineral and textural analyses were then carried out at Central Department of Geology (CDG), Tribhuvan University, Kirtipur. The abrasion test was carried out at Turbine Testing Lab (TTL), Department of Mechanical Engineering college, Kathmandu University, Dhulikhel, Kavrepalanchok District.

Two sets of Glass slides were prepared for two separate size-fractions, i.e., 500-250 $\mathrm{mm}$ and $250 \mathrm{~mm}-$ Pan for each of the samples. One of the sets was stained for feldspars after Hayes and Klugman (1959), and the remaining set was stained for carbonate minerals. In the latter, a sample slide was immersed in silver nitrate solution for 3-4 minutes, and after that the slide was immersed in potassium chromate for 1 minute. By this method, calcite and aragonite are stained deep red-brown and dolomite is stained faint to light brown.

Shapes of particles were observed under a binocular stereo zoom microscope (Olympus SZ) to identify roundness using a Power's (1953) chart, and sphericity using a Rittenhouse's (1943) chart. Mineral composition is an important parameter to define river sediment. Hardness of mineral is one most important criterion to determine its erosion potential. Those minerals which have Moho's hardness scale greater than that of turbine material can erode the runner material. On average turbine materials have Moho's hardness of 6-6.5 depending on its composition and coatings. Thus, all the minerals having
Moho's hardness greater than 6 value has potential to scratch turbine. Quartz (H7) and feldspar (H6) are the most common minerals in river sediment which can potentially cause erosion of turbine. To perform mineral analysis, Gazzi-Dickinson grain counting method (Ingersoll et al., 1984) was followed as this method is cost efficient and easier to use although it is time consuming. Then the hardness index was calculated as the ratio of the product of number of specific mineral and its corresponding hardness to the total number of minerals counted.

\section{Test on Rotating Disc Apparatus (RDA)}

Rotating Disc Apparatus (RDA) (Fig. 2) was designed and fabricated at Kathmandu University at Turbine Testing Laboratory (TTL) in Kathmandu University (KU), Nepal (Rajkarnikar, 2013). This was designed to carry out tests of sediment erosion in Francis runner blades. This apparatus consists of a rotating disc, motor, shaft, etc. The rotor assembly consists of the disc attached with a shaft. The disc has a square hole in the center, which fits with the male part of the shaft. The inner chamber is the cooling chamber whereas the outer chamber encloses the rotating disc, test specimens and the sand water mixture. Test specimens are screwed on rotating disc.

There is no standard specification for making sediment and water concentration because it is too complex to make real environment of actual flow condition, operating time, variation of size and concentration, impingement angle, head, and material (Padhy et al., 2008). Thus, this research is based on relative abrasion by sample according to location wise. Test specimen was made on workshop as shown in Fig. 3 and then it was grinded and painted with four layers of different enamel color. At first blue color was applied and then red, yellow and green, respectively. Removal of paint shows the abrasion potential of sediment.

The rotating disc was placed in a closed housing with water and sediment and driven by motor upto the speed of 1440 rpm. Initial weight of specimen and operation time was noted. Patterns of erosion on the blades were observed by removal of painted surface on the blade after an operation time of 30 minutes. About 900 grams of sand sample were mixed up with nine liters of water and were used to test for 30 minutes. After finished rotating, the test specimens were unmounted from the rotating disc, washed, dried and were weighed again. Consecutively, two specimens were used to perform one test for each location. The rate of removal of material due to sediment erosion was estimated by the measurement of weight loss from the blades after successive test.

\section{RESULTS}

\section{Texture}

Sediment samples from the Budhi Gandaki, Trishuli, Marsyangdi, Seti rivers were characterized by roundness and sphericity (Table 1). Results show that the size smaller than 


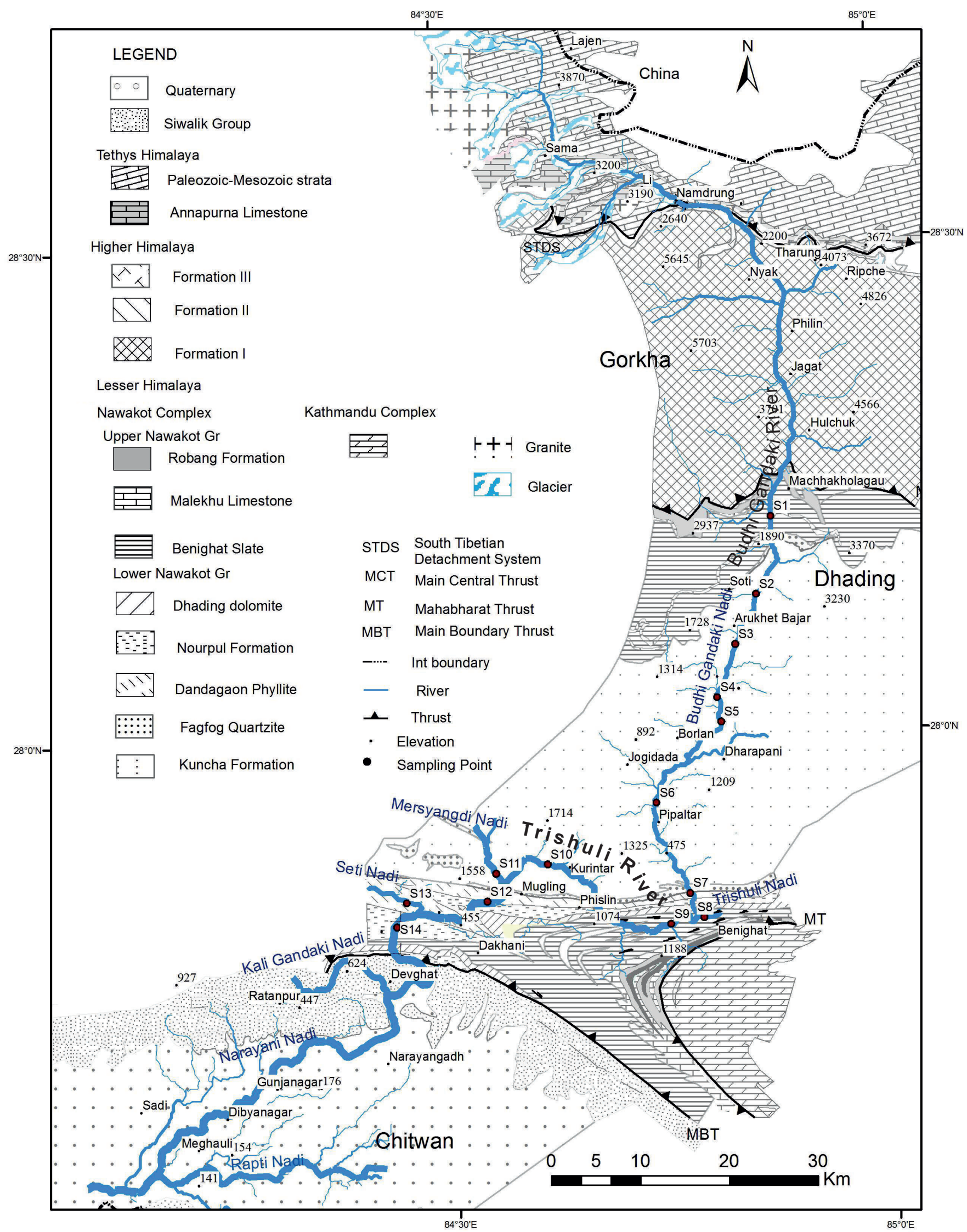

Fig. 1: Map with sampling numbers 


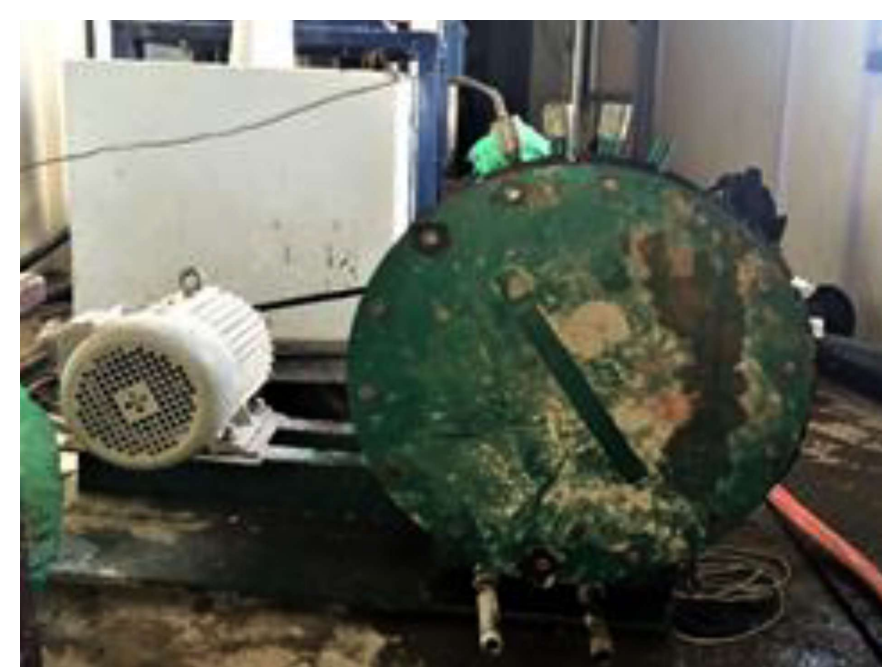

Fig. 2: Rotating Disc Apparatus (Specification: Power Source: 3 phase Variable frequency drive; Motor: 2.2 KW; Ampere: 5.03; Motor speed: $1440 \mathrm{rpm}$; Disc speed: 2880 rpm; Step up drive: Toothed belt drive; Cooling: External Circulating water)

250 microns has sub angular grains. Samples S2 (Soti) and S5 (Rampur tar) have mean roundness 1.98 and 1.83, respectively indicating angular grains. From the Budhi Gandaki River, sample S4 has the highest value of mean roundness 2.22 (Sub angular). Mean sphericity ranges from 0.70 to 0.71 . For samples S8-C1 (Trishuli), S11-C2 (Marsyangdi) and S13-C13 (Seti) Rivers, the mean roundness values are 2.12, 2.11 and 2.04, respectively (Table 1) showing the dominance of sub angular grain shape in these areas. Mean sphericity of these rivers sample are 0.68 , 0.71 and 0.72 respectively.

Results exhibit that particles having size between 250 -500 microns are predominantly sub angular. Sample S5 has mean roundness 1.99 and mean sphericity 0.72 . Mean sphericity ranges from 0.71 to 0.76 . Sample S8-C1 (Trishuli), S11-C2 (Marsyangdi) and S13-C13 (Seti) River have mean roundness of 2.4, 1.99 and 2.15, respectively indicating sub angular grain shape. Mean sphericity of these rivers sample are $0.73,0.75$ and 0.74 , respectively.

\section{Mineral composition}

Quartz is found to be the common mineral in river sediments (proportion is more than $50 \%$ in all samples) (Table 2). It has Mohr's hardness of 7 and hence can easily wears turbines. For size $<250$ microns, sample S5 has highest percentage $(62.95 \%)$ and S4 has least percentage $(54.11 \%)$ of quartz (Table 2). Sample S2 has the highest (15.16\%) and S3 has the least $(9.32 \%)$ percentage of feldspar. Sample S5 has the highest value of hardness index (6.072) followed by sample S8-C1: Trishuli (6.009) (Table 2). Sample S4 has the lowest hardness index value (5.718) among all samples. At the confluence of rivers, S8-C1 (Trishuli), S11-C2 (Marsyangdi) and S13-C3 (Seti), rivers have 57.83 \%, 56.52\% and 54.69\% quartz, $15.33 \%, 13.04 \%$ and $8.98 \%$ feldspar. Samples collected from the Trishuli River (S8-C1), Marsyangdi river (S11-C2) and the Seti river (S13-C3) have respectively 6.009, 5.840 and 5.749 hardness index respectively.

For grain size 250-500 microns, sample S3 and S12 have respectively $61.86 \%$ and $52.54 \%$ quartz (Table 2 ). Sample S10 has the highest (18.37\%) and S3 lowest (9.30\%) feldspar. Sample S6 has the highest value of hardness index (6.088) (Table 3). Sample S14 has the lowest hardness index (5.696) among all the samples. In the confluence rivers S8-C1 (Trishuli), S11-C2 (Marsyangdi) and S13-C3 (Seti) quartz is respectively, $58.02 \%, 58.08 \%$ and $53.82 \%$, and feldspar is $14.88 \%, 9.19$ $\%$ and $16.47 \%$ feldspar. Samples from the Trishuli River (S8C1), Marsyangdi River (S11-C2) and the Seti River (S13-C3) have hardness index respectively 6.019, 5.821 and 5.820).

Table 1: mean roundness and mean sphericity of fraction size $(250 \mu \mathrm{m}-$ pan $)$ and $(500 \mu \mathrm{m}-250 \mu \mathrm{m})$

\begin{tabular}{|c|c|c|c|c|c|c|}
\hline \multirow[b]{2}{*}{ Sample no. } & \multicolumn{3}{|c|}{$250 \mu \mathrm{m}-$ pan } & \multicolumn{3}{|c|}{$500 \mu \mathrm{m}-250 \mu \mathrm{m}$} \\
\hline & $\mathrm{Me}$ & ndness & Mean sphericity & Mea & & Mean sphericity \\
\hline S1 & 2.01 & $\mathrm{SA}$ & 0.72 & 2.02 & $\mathrm{SA}$ & 0.73 \\
\hline S2 & 1.98 & A & 0.71 & 2.03 & SA & 0.71 \\
\hline S3 & 2.1 & SA & 0.73 & 2.2 & SA & 0.73 \\
\hline $\mathrm{S} 4$ & 2.22 & SA & 0.75 & 2.24 & SA & 0.74 \\
\hline S5 & 1.83 & A & 0.7 & 1.99 & A & 0.72 \\
\hline S6 & 2.09 & SA & 0.75 & 2.17 & SA & 0.75 \\
\hline S7 & 2.21 & SA & 0.72 & 2.25 & SA & 0.76 \\
\hline S9 & 2.07 & SA & 0.72 & 2.05 & SA & 0.76 \\
\hline $\mathrm{S} 10$ & 2.14 & SA & 0.73 & 2.5 & SA & 0.74 \\
\hline $\mathrm{S} 12$ & 2.19 & SA & 0.73 & 2.13 & SA & 0.73 \\
\hline S14 & 2.4 & SA & 0.74 & 2.41 & SA & 0.75 \\
\hline S8-C1 Trishuli River & 2.12 & SA & 0.68 & 2.4 & SA & 0.73 \\
\hline $\begin{array}{l}\text { S11-C2 Marsyangdi } \\
\text { River }\end{array}$ & 2.11 & SA & 0.71 & 1.99 & A & 0.75 \\
\hline S13-C3 Seti River & 2.04 & SA & 0.72 & 2.15 & SA & 0.74 \\
\hline
\end{tabular}


Table 2: Minerals composition of fine aggregates from the Budhi Gandaki-Trishuli River

\begin{tabular}{|c|c|c|c|c|c|c|c|c|c|c|c|c|c|c|c|c|c|c|c|c|}
\hline \multirow{2}{*}{ Sample no } & \multirow[b]{2}{*}{$\mathrm{Q}$} & \multicolumn{3}{|c|}{ Feldspar } & \multicolumn{6}{|c|}{ Fine Lithic Fragments } & \multicolumn{4}{|c|}{$\begin{array}{l}\text { Coarse Lithic } \\
\text { Fragments }\end{array}$} & \multirow{2}{*}{ 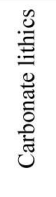 } & \multicolumn{3}{|c|}{ Mica } & \multirow[b]{2}{*}{ 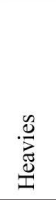 } & \multirow[b]{2}{*}{ 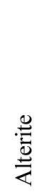 } \\
\hline & & $\mathrm{Pl}$ & $\mathrm{KF}$ & 吾 & $\begin{array}{l}\vec{n} \\
n_{n}\end{array}$ & $\frac{\frac{\omega}{\pi}}{\frac{\pi}{\omega}}$ & $\begin{array}{l}\frac{\tilde{n}}{\overline{0}} \\
\dot{n}\end{array}$ & 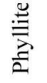 & $\frac{\ddot{v}}{\omega}$ & 퓽 & $\sum_{d}^{I}$ & $\sum_{ \pm}$ & $\stackrel{+1}{d}$ & స్ & & 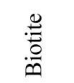 & 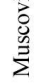 & 퓽 & & \\
\hline \multicolumn{21}{|c|}{$500 \mu \mathrm{m}-250 \mu \mathrm{m}$} \\
\hline $\mathrm{S} 1$ & 59.7 & 7.2 & 3.1 & 10.3 & 0.3 & 0 & 1.4 & 2.1 & 0 & 3.8 & 6.6 & 0.7 & 0.7 & 7.9 & 0.3 & 12.1 & 4.8 & 17 & 0 & 1 \\
\hline $\mathrm{S} 2$ & 58.5 & 11 & 4 & 15.2 & 1.4 & 0 & 1.1 & 2.5 & 0 & 5.1 & 5.8 & 0.7 & 0.7 & 7.2 & 0.4 & 7.58 & 5.4 & 13 & 0.4 & 0.4 \\
\hline S3 & 57.7 & 6.5 & 2.9 & 9.32 & 1.1 & 0.7 & 1.1 & 1.4 & 0 & 4.3 & 8.2 & 1.4 & 0 & 9.7 & 0.7 & 10.4 & 5.7 & 16 & 0.7 & 1.4 \\
\hline $\mathrm{S} 4$ & 54.1 & 10 & 3.9 & 14 & 0.5 & 0 & 3.4 & 1.9 & 0 & 5.8 & 6.3 & 0.5 & 0 & 6.8 & 0 & 13 & 6.3 & 19 & 0 & 0 \\
\hline S5 & 63 & 6.2 & 4.9 & 11.2 & 1.6 & 0 & 1.6 & 0.7 & 0.3 & 4.3 & 4.9 & 1.6 & 0 & 6.6 & 0 & 10.2 & 3.9 & 14 & 0 & 1 \\
\hline S6 & 57 & 7.2 & 3.3 & 10.5 & 1.4 & 0 & 1.4 & 2.9 & 0 & 5.8 & 7.9 & 0.4 & 0.4 & 8.7 & 0.4 & 9.02 & 6.1 & 15 & 0 & 2.5 \\
\hline S7 & 58.6 & 6.1 & 3.7 & 9.77 & 0.9 & 0.5 & 2.3 & 1.4 & 0 & 5.1 & 7.9 & 0 & 0.5 & 8.4 & 0.9 & 8.84 & 7.4 & 16 & 0.9 & 0 \\
\hline S9 & 55.8 & 10 & 4.7 & 14.9 & 1.1 & 0.4 & 1.8 & 2.5 & 0 & 5.8 & 6.9 & 1.1 & 0 & 8 & 0 & 9.05 & 5.8 & 15 & 0.4 & 0.4 \\
\hline $\mathrm{S} 10$ & 54.4 & 9.5 & 5.6 & 15.1 & 1.4 & 0 & 1.8 & 2.5 & 0 & 5.6 & 8.1 & 1.4 & 0.7 & 10 & 0 & 9.12 & 3.9 & 13 & 0 & 1.8 \\
\hline $\mathrm{S} 12$ & 56.5 & 10 & 2.7 & 12.7 & 0 & 0.4 & 1.5 & 1.9 & 0 & 3.8 & 6.2 & 0.8 & 0.4 & 7.3 & 0.4 & 9.62 & 6.2 & 16 & 0.4 & 3.1 \\
\hline $\mathrm{S} 14$ & 55.6 & 8.4 & 7.1 & 15.6 & 1.3 & 0 & 1.8 & 2.7 & 0 & 5.8 & 3.6 & 0.9 & 0 & 4.4 & 0 & 14.2 & 4 & 18 & 0 & 0.4 \\
\hline S8-C1 (Trishuli) & 57.8 & 10 & 5.1 & 15.3 & 1.9 & 0 & 1.6 & 1.3 & 0 & 4.8 & 6.7 & 0 & 0 & 6.7 & 0.3 & 8.94 & 4.5 & 13 & 0 & 1.6 \\
\hline S11-C2 (Marsyangdi) & 56.5 & 9.2 & 3.8 & 13 & 2.2 & 0 & 2.2 & 4.4 & 0 & 8.7 & 4.4 & 1.1 & 0.5 & 6 & 0 & 9.24 & 4.4 & 14 & 0 & 2.2 \\
\hline S13-C3 (Seti) & 54.7 & 7 & 2 & 8.98 & 1.2 & 0 & 2.3 & 1.6 & 0.4 & 5.5 & 8.2 & 1.6 & 0.4 & 10 & 0.4 & 12.1 & 7 & 19 & 0.4 & 0.8 \\
\hline \multicolumn{21}{|c|}{$500 \mu \mathrm{m}-250 \mu \mathrm{m}$} \\
\hline S1 & 60.6 & 9.1 & 3.5 & 12.6 & 2.6 & 0.4 & 1.3 & 1.3 & 0 & 5.6 & 3.5 & 0.4 & 0 & 3.9 & 0.00 & 10.38 & 5.2 & 16 & 0.4 & 1.3 \\
\hline $\mathrm{S} 2$ & 59.4 & 7.9 & 5.3 & 13.2 & 0.8 & 0 & 0.4 & 2.3 & 0 & 3.4 & 4.5 & 0.8 & 0 & 5.3 & 0.00 & 12.03 & 5.3 & 17 & 0.4 & 1.1 \\
\hline S3 & 61.9 & 6.5 & 2.8 & 9.3 & 0.9 & 0 & 0.9 & 2.3 & 0 & 4.2 & 6.5 & 0 & 0.5 & 7 & 0.93 & 9.30 & 3.7 & 13 & 0.9 & 2.8 \\
\hline S4 & 56.8 & 9 & 3.2 & 12.2 & 1.8 & 0.4 & 2.2 & 1.4 & 0.4 & 6.1 & 6.5 & 2.2 & 0 & 8.6 & 0.72 & 9.71 & 3.2 & 13 & 0.4 & 2.2 \\
\hline S5 & 59.8 & 6.5 & 5.4 & 11.9 & 1.2 & 0.4 & 1.9 & 1.2 & 0 & 4.6 & 4.2 & 1.2 & 0.8 & 6.1 & 0.00 & 10.73 & 6.9 & 18 & 0 & 0 \\
\hline S6 & 60.2 & 8.1 & 4.1 & 12.2 & 1.5 & 0.4 & 1.5 & 1.1 & 0 & 4.4 & 7.8 & 2.2 & 0 & 10 & 0.00 & 8.86 & 3 & 12 & 0 & 1.5 \\
\hline S7 & 59.3 & 7.7 & 4.8 & 12.5 & 0.4 & 0 & 1.6 & 2.4 & 0 & 4.4 & 4.4 & 0.8 & 0 & 5.2 & 0.00 & 9.68 & 5.2 & 15 & 0.4 & 3.2 \\
\hline S9 & 56.8 & 11 & 4.8 & 16.1 & 1.8 & 0.4 & 1.5 & 2.6 & 0 & 6.2 & 5.9 & 0.4 & 0 & 6.2 & 0.37 & 8.42 & 5.1 & 14 & 0 & 0.7 \\
\hline $\mathrm{S} 10$ & 54.8 & 13 & 5.4 & 18.4 & 2.4 & 0 & 2.7 & 1.7 & 0.3 & 7.1 & 6.1 & 0 & 0 & 6.1 & 0.00 & 8.84 & 3.7 & 13 & 0.7 & 0.3 \\
\hline $\mathrm{S} 12$ & 52.5 & 6.5 & 5.1 & 11.6 & 1.1 & 0 & 1.1 & 3.3 & 0 & 5.4 & 9.1 & 2.2 & 0.7 & 12 & 0.00 & 11.96 & 4.7 & 17 & 0.4 & 1.5 \\
\hline $\mathrm{S} 14$ & 55.4 & 6.1 & 3.9 & 9.96 & 2.2 & 0.4 & 3 & 3.5 & 0 & 9.1 & 6.1 & 0 & 0 & 6.1 & 0.00 & 11.25 & 6.9 & 18 & 0.4 & 0.9 \\
\hline S8-C1 (Trishuli) & 58 & 11 & 3.8 & 14.9 & 1.9 & 0.4 & 0 & 2.3 & 0 & 4.6 & 5.3 & 1.9 & 0 & 7.3 & 0.76 & 8.01 & 4.2 & 12 & 0.8 & 1.5 \\
\hline S11-C2 (Marsyangdi) & 58.1 & 6.6 & 2.6 & 9.19 & 1.8 & 0.7 & 1.5 & 2.9 & 0 & 7 & 5.9 & 2.2 & 0 & 8.1 & 0.00 & 12.50 & 4 & 17 & 0 & 1.1 \\
\hline S13-C3 (Seti) & 53.8 & 11 & 5.3 & 16.5 & 1.5 & 0 & 2.7 & 3.5 & 0 & 7.7 & 5.3 & 1.2 & 0.6 & 7.1 & 0.29 & 8.23 & 6.2 & 14 & 0.3 & 0 \\
\hline
\end{tabular}

Table 3: Hardness index of mineral constituents of fine aggregates

\begin{tabular}{|c|c|c|c|c|c|c|c|c|c|c|c|c|c|c|c|c|}
\hline Sample no. & $\begin{array}{l}n \\
\% \\
\circ \\
0\end{array}$ & $\begin{array}{l}\text { Feldspar } \\
\text { Total * } \\
6\end{array}$ & 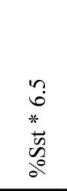 & $\begin{array}{l}m \\
* \\
\frac{0}{\pi} \\
\frac{\pi}{\tilde{n}} \\
0\end{array}$ & $\begin{array}{l}n \\
m \\
* \\
. \tilde{n} \\
\frac{n}{0} \\
0 \\
0 \\
0\end{array}$ & 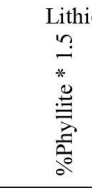 & $\begin{array}{l}n \\
n \\
* \\
\frac{0}{0} \\
\frac{0}{5} \\
0\end{array}$ & 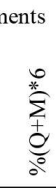 & $\sum_{\sum_{0}^{+}}^{n}$ & 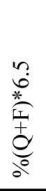 & 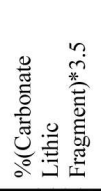 & 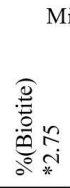 & 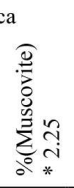 & 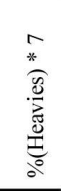 & 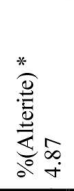 & 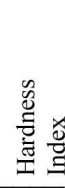 \\
\hline \multicolumn{17}{|c|}{$250 \mu \mathrm{m}-$ pan } \\
\hline S1 & 417.6 & 62.0 & 2.2 & 0.0 & 4.8 & 3.1 & 0.0 & 39.3 & 3.7 & 4.4 & 1.2 & 33.2 & 10.6 & 0.0 & 5.0 & 5.9 \\
\hline S2 & 409.4 & 91.0 & 9.4 & 0.0 & 3.8 & 3.8 & 0.0 & 34.7 & 4.0 & 4.7 & 1.3 & 20.9 & 11.9 & 2.5 & 1.8 & 6.0 \\
\hline S3 & 404.0 & 55.9 & 7.0 & 2.2 & 3.8 & 2.2 & 0.0 & 49.4 & 7.9 & 0.0 & 2.5 & 28.6 & 12.6 & 5.0 & 7.0 & 5.9 \\
\hline S4 & 378.8 & 84.1 & 3.1 & 0.0 & 11.8 & 2.9 & 0.0 & 37.7 & 2.6 & 0.0 & 0.0 & 35.9 & 13.8 & 0.0 & 0.0 & 5.7 \\
\hline S5 & 440.7 & 66.9 & 10.7 & 0.0 & 5.7 & 1.0 & 1.8 & 29.5 & 9.0 & 0.0 & 0.0 & 27.9 & 8.7 & 0.0 & 4.8 & 6.1 \\
\hline S6 & 399.3 & 62.8 & 9.4 & 0.0 & 5.0 & 4.3 & 0.0 & 47.6 & 2.0 & 2.3 & 1.3 & 24.8 & 13.5 & 0.0 & 12.3 & 5.9 \\
\hline S7 & 410.2 & 58.6 & 6.1 & 1.4 & 8.2 & 2.1 & 0.0 & 47.5 & 0.0 & 3.0 & 3.3 & 24.3 & 16.4 & 6.5 & 0.0 & 5.9 \\
\hline S9 & 390.5 & 89.1 & 7.0 & 1.1 & 6.3 & 3.8 & 0.0 & 41.3 & 5.9 & 0.0 & 0.0 & 24.9 & 12.7 & 2.5 & 1.8 & 5.9 \\
\hline S10 & 380.7 & 90.5 & 9.1 & 0.0 & 6.1 & 3.7 & 0.0 & 48.4 & 7.7 & 4.6 & 0.0 & 25.1 & 8.5 & 0.0 & 8.5 & 5.9 \\
\hline $\mathrm{S} 12$ & 395.8 & 76.1 & 0.0 & 1.1 & 5.4 & 2.9 & 0.0 & 36.9 & 4.2 & 2.5 & 1.3 & 26.5 & 13.5 & 2.7 & 15.0 & 5.8 \\
\hline S14 & 388.9 & 93.4 & 8.7 & 0.0 & 6.2 & 4.0 & 0.0 & 21.4 & 4.9 & 0.0 & 0.0 & 39.1 & 8.8 & 0.0 & 2.1 & 5.8 \\
\hline S8-C1 (Trishuli River) & 404.8 & 92.0 & 12.5 & 0.0 & 5.6 & 1.9 & 0.0 & 40.3 & 0.0 & 0.0 & 1.1 & 24.6 & 9.8 & 0.0 & 7.7 & 6.0 \\
\hline S11-C2 (Marsyangdi River) & 395.6 & 78.2 & 14.1 & 0.0 & 7.6 & 6.5 & 0.0 & 26.1 & 5.9 & 3.5 & 0.0 & 25.4 & 9.6 & 0.0 & 10.6 & 5.8 \\
\hline S13-C3 (Seti River) & 382.8 & 53.9 & 7.6 & 0.0 & 8.2 & 2.3 & 2.2 & 49.2 & 8.6 & 2.5 & 1.4 & 33.3 & 15.5 & 2.7 & 3.8 & 5.7 \\
\hline \multicolumn{17}{|c|}{$500 \mu \mathrm{m}-250 \mu \mathrm{m}$} \\
\hline S1 & 424.3 & 75.3 & 16.8 & 1.3 & 4.5 & 1.9 & 0.0 & 20.8 & 2.4 & 0.0 & 0.0 & 28.6 & 11.4 & 3.0 & 6.3 & 6.0 \\
\hline S2 & 415.7 & 79.0 & 4.9 & 0.0 & 1.3 & 3.4 & 0.0 & 27.1 & 4.1 & 0.0 & 0.0 & 33.1 & 11.6 & 2.6 & 5.5 & 5.9 \\
\hline S3 & 433.0 & 55.8 & 6.1 & 0.0 & 3.3 & 3.5 & 0.0 & 39.1 & 0.0 & 3.0 & 3.3 & 25.6 & 8.2 & 6.5 & 13.6 & 6.0 \\
\hline S4 & 397.8 & 73.4 & 11.6 & 1.1 & 7.6 & 2.2 & 2.0 & 38.8 & 11.9 & 0.0 & 2.5 & 26.7 & 7.1 & 2.5 & 10.5 & 6.0 \\
\hline S5 & 418.4 & 71.2 & 7.5 & 1.1 & 6.7 & 1.7 & 0.0 & 25.3 & 6.3 & 5.0 & 0.0 & 29.5 & 15.2 & 0.0 & 0.0 & 5.9 \\
\hline S6 & 421.1 & 73.1 & 9.6 & 1.1 & 5.2 & 1.7 & 0.0 & 46.5 & 12.2 & 0.0 & 0.0 & 24.4 & 6.5 & 0.0 & 7.2 & 6.1 \\
\hline S7 & 414.9 & 75.0 & 2.6 & 0.0 & 5.6 & 3.6 & 0.0 & 26.6 & 4.4 & 0.0 & 0.0 & 26.6 & 11.5 & 2.8 & 15.7 & 5.9 \\
\hline S9 & 397.5 & 96.7 & 11.9 & 1.1 & 5.1 & 3.8 & 0.0 & 35.2 & 2.0 & 0.0 & 1.3 & 23.2 & 11.3 & 0.0 & 3.6 & 5.9 \\
\hline S10 & 383.3 & 110.2 & 15.5 & 0.0 & 9.5 & 2.6 & 1.9 & 36.7 & 0.0 & 0.0 & 0.0 & 24.3 & 8.2 & 4.8 & 1.7 & 6.0 \\
\hline $\mathrm{S} 12$ & 367.8 & 69.5 & 7.1 & 0.0 & 3.8 & 4.9 & 0.0 & 54.4 & 11.9 & 4.7 & 0.0 & 32.9 & 10.4 & 2.5 & 7.1 & 5.8 \\
\hline S14 & 387.9 & 59.8 & 14.0 & 1.3 & 10.6 & 5.2 & 0.0 & 36.4 & 0.0 & 0.0 & 0.0 & 30.9 & 15.3 & 3.0 & 4.2 & 5.7 \\
\hline S8-C1 (Trishuli River) & 406.1 & 89.3 & 12.4 & 1.1 & 0.0 & 3.4 & 0.0 & 32.0 & 10.5 & 0.0 & 2.7 & 22.0 & 9.2 & 5.3 & 7.5 & 6.0 \\
\hline S11-C2 (Marsyangd River) & 406.6 & 55.1 & 12.0 & 2.2 & 5.2 & 4.4 & 0.0 & 35.3 & 12.2 & 0.0 & 0.0 & 34.4 & 8.9 & 0.0 & 5.4 & 5.8 \\
\hline S13-C3 (Seti River) & 376.7 & 98.8 & 9.6 & 0.0 & 9.3 & 5.3 & 0.0 & 31.7 & 6.5 & 3.8 & 1.0 & 22.6 & 13.6 & 2.0 & 0.0 & 5.8 \\
\hline
\end{tabular}




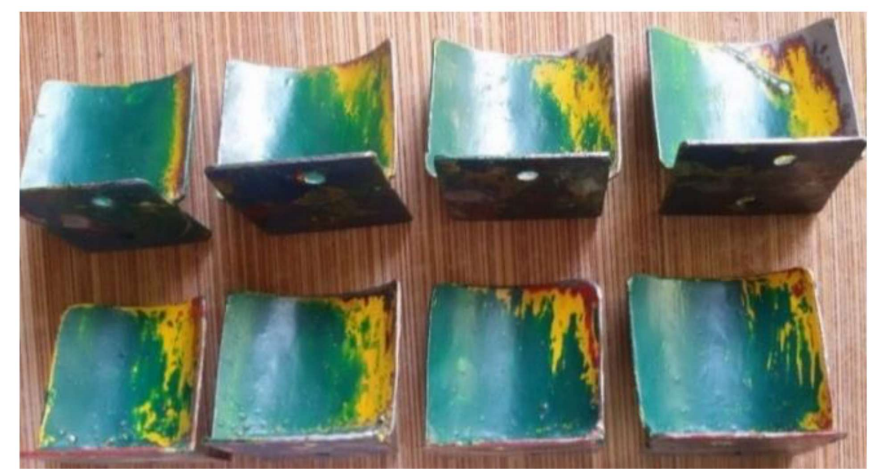

Fig. 3: Test specimen after abrasion test

\section{Abrasion test results}

Here two specimens were used to test in each test. Initial and final weights of every specimen was noted. Material, concentration and time were made constant to conduct the test. Test specimens after abration were collected (Fig. 3) and weight loss in percentage was calculated, and the average weight loss and abrasion rate from two test specimens were calculated after the equation of Rajkarnikar (2013).
Abrasion rate $=\left[\left(\mathrm{W}_{\mathrm{i}}-\mathrm{W}_{\mathrm{f}}\right) /\left(\mathrm{W}_{\mathrm{i}} * \mathrm{~d}_{\mathrm{t}}\right)\right] .1000(\mathrm{mg} / \mathrm{g} /$ hour $)$

Where, $\mathrm{W}_{\mathrm{i}}=$ initial weight of a specimen

$\mathrm{W}_{\mathrm{f}}=$ final weight of a specimen

In 250 microns-pan fraction size, S5 at the Rampur Tar has the highest abrasion rate $7.57 \mathrm{mg} / \mathrm{g}$ per hour with weight loss $248 \mathrm{mg}$ (Table 4; Fig. 4). This is followed by second highest S2 at Soti with abrasion rate $7.43 \mathrm{mg} / \mathrm{g}$ per hour with weight loss $237.5 \mathrm{mg}$. In this fraction size S12 has a low abrasion rate $5.38 \mathrm{mg} / \mathrm{g}$ per hour with $171.5 \mathrm{mg}$. Among the confluence rivers, the Trishuli River has high abrasion rate $6.94 \mathrm{mg} / \mathrm{g}$ per hour with weight loss of $226 \mathrm{mg}$.

In 500-250-micron size fraction, S5 at the Rampur Tar has the highest abrasion rate $13.75 \mathrm{mg} / \mathrm{g}$ per hour with weight loss $398.5 \mathrm{mg}$ (Table 4; Fig. 4). This is followed by second highest S1 with abrasion rate $13.35 \mathrm{mg} / \mathrm{g}$ per hour with weight loss $395 \mathrm{mg}$. Sample S14 at the Ghumaune has low abrasion rate $10.80 \mathrm{mg} / \mathrm{g}$ per hour with weight loss of $311 \mathrm{mg}$. Among the confluence rivers, the Trishuli River has high abrasion rate $11.97 \mathrm{mg} / \mathrm{g}$ per hour with weight loss $352 \mathrm{mg}$.

Table 4: Result of abrasion loss and rate of abrasion of a rotating disc by fine aggregate

\begin{tabular}{|c|c|c|c|c|c|c|c|c|c|}
\hline \multirow[b]{2}{*}{ Sample No. } & & \multicolumn{4}{|c|}{$250 \mu \mathrm{m}-$ pan } & \multicolumn{4}{|c|}{$500 \mu \mathrm{m}-250 \mu \mathrm{m}$} \\
\hline & & $\begin{array}{l}\text { Initial } \\
\text { weight } \\
\left(\mathrm{W}_{\mathrm{i}}\right) \mathrm{g}\end{array}$ & $\begin{array}{l}\text { Final } \\
\text { weight } \\
\left(W_{f}\right) g\end{array}$ & $\begin{array}{l}\text { Initial-final } \\
\text { weight, } \Delta \mathrm{m} \\
=\left(\mathrm{W}_{\mathrm{i}}-\mathrm{W}_{\mathrm{f}}\right)\end{array}$ & $\begin{array}{c}\text { Average } \\
\text { abrasion rate } \\
\text { (mg/g per hour) }\end{array}$ & $\begin{array}{l}\text { Initial } \\
\text { weight } \\
\left(\mathrm{W}_{\mathrm{i}}\right) \mathrm{g}\end{array}$ & $\begin{array}{l}\text { Final } \\
\text { weight } \\
\left(W_{f}\right) g\end{array}$ & $\begin{array}{l}\text { Initial-final } \\
\text { weight, } \Delta \mathrm{m} \\
=\left(\mathrm{W}_{\mathrm{i}}-\mathrm{W}_{\mathrm{f}}\right)\end{array}$ & $\begin{array}{c}\text { Average } \\
\text { abrasion rate } \\
(\mathrm{mg} / \mathrm{g} \text { per hour })\end{array}$ \\
\hline \multirow[t]{2}{*}{ S1 } & $\mathrm{a}$ & 63.75 & 63.503 & 0.249 & 7.22 & 59.345 & 58.94 & 0.404 & 13.35 \\
\hline & $\mathrm{b}$ & 63.75 & 63.540 & 0.211 & & 59.023 & 58.64 & 0.386 & \\
\hline \multirow[t]{2}{*}{ S2 } & a & 64.26 & 64.023 & 0.236 & 7.43 & 55.265 & 54.92 & 0.347 & 12.02 \\
\hline & $\mathrm{b}$ & 63.57 & 63.326 & 0.239 & & 57.312 & 56.98 & 0.329 & \\
\hline \multirow[t]{2}{*}{ S3 } & a & 64.66 & 64.437 & 0.218 & 6.63 & 57.344 & 56.99 & 0.358 & 11.81 \\
\hline & $\mathrm{b}$ & 63.87 & 63.663 & 0.208 & & 56.272 & 55.96 & 0.313 & \\
\hline \multirow[t]{2}{*}{ S4 } & a & 63.31 & 63.119 & 0.193 & 6.19 & 58.782 & 58.45 & 0.328 & 11.41 \\
\hline & $\mathrm{b}$ & 62.95 & 62.752 & 0.198 & & 57.413 & 57.08 & 0.335 & \\
\hline \multirow[t]{2}{*}{ S5 } & a & 65.43 & 65.191 & 0.241 & 7.57 & 56.287 & 55.88 & 0.409 & 13.75 \\
\hline & $\mathrm{b}$ & 65.56 & 65.306 & 0.255 & & 59.839 & 59.45 & 0.388 & \\
\hline \multirow[t]{2}{*}{ S6 } & a & 62.41 & 62.212 & 0.196 & 6.69 & 54.583 & 54.23 & 0.358 & 12.17 \\
\hline & $\mathrm{b}$ & 64.17 & 63.937 & 0.228 & & 54.039 & 53.74 & 0.303 & \\
\hline \multirow[t]{2}{*}{ S7 } & $\mathrm{a}$ & 62.63 & 62.397 & 0.237 & 7.09 & 54.142 & 53.79 & 0.351 & 12.39 \\
\hline & $\mathrm{b}$ & 62.95 & 62.737 & 0.208 & & 56.892 & 56.56 & 0.336 & \\
\hline \multirow[t]{2}{*}{ S9 } & $\mathrm{a}$ & 63.32 & 63.133 & 0.184 & 5.94 & 58.029 & 57.68 & 0.345 & 11.59 \\
\hline & $\mathrm{b}$ & 65.61 & 65.414 & 0.199 & & 59.587 & 59.23 & 0.359 & \\
\hline \multirow[t]{2}{*}{ S10 } & $\mathrm{a}$ & 64.64 & 64.393 & 0.243 & 6.52 & 53.175 & 52.85 & 0.323 & 11.03 \\
\hline & $\mathrm{b}$ & 63.71 & 63.538 & 0.176 & & 54.218 & 53.92 & 0.299 & \\
\hline \multirow[t]{2}{*}{$\mathrm{S} 12$} & $\mathrm{a}$ & 64.28 & 64.119 & 0.158 & 5.38 & 56.415 & 56.1 & 0.317 & 11.75 \\
\hline & $\mathrm{b}$ & 63.34 & 63.154 & 0.185 & & 57.633 & 57.32 & 0.312 & \\
\hline \multirow[t]{2}{*}{ S14 } & $\mathrm{a}$ & 63.39 & 63.192 & 0.198 & 5.77 & 54.394 & 54.06 & 0.333 & 10.8 \\
\hline & $\mathrm{b}$ & 64.62 & 64.445 & 0.171 & & 56.594 & 56.28 & 0.317 & \\
\hline \multirow{2}{*}{$\begin{array}{l}\text { S8-Cl (Trishuli } \\
\text { River) }\end{array}$} & $\mathrm{a}$ & 63.92 & 63.686 & 0.237 & 6.94 & 55.878 & 55.56 & 0.315 & 11.97 \\
\hline & $\mathrm{b}$ & 66.59 & 66.378 & 0.215 & & 56.258 & 55.91 & 0.344 & \\
\hline \multirow{2}{*}{$\begin{array}{l}\text { S11-C2 } \\
\text { (Marsyangdi River) }\end{array}$} & a & 64.5 & 64.228 & 0.274 & 6.22 & 59.124 & 58.82 & 0.301 & 11.72 \\
\hline & $\mathrm{b}$ & 64.29 & 64.165 & 0.127 & & 53.018 & 52.69 & 0.326 & \\
\hline \multirow[t]{2}{*}{ S13-C3 (Seti River) } & $\mathrm{a}$ & 66.14 & 65.937 & 0.206 & 6.53 & 58.926 & 58.62 & 0.309 & 11.24 \\
\hline & $\mathrm{b}$ & 64.74 & 64.523 & 0.221 & & 56.311 & 56 & 0.313 & \\
\hline
\end{tabular}




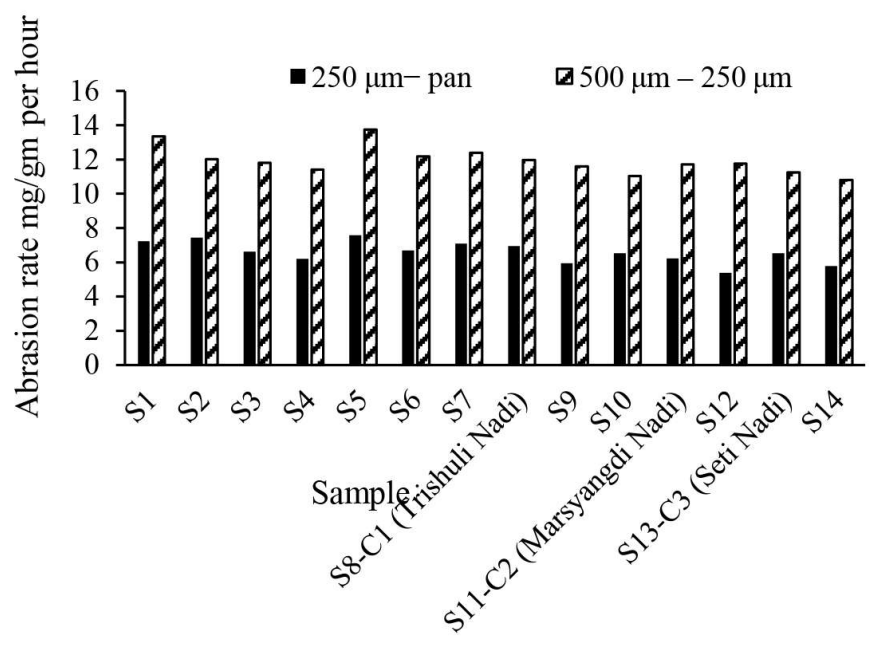

Fig. 4: Bar diagram of Erosion test

When abrasivity was plotted against mean roundness, mean sphericity, \%quartz and harness index (Figs. 5 and 6), the trend lines show that the abrasivity decreases with the increase of roundness and sphericity of grains but increases with the increase of $\%$ of quartz and hardness index of the samples.

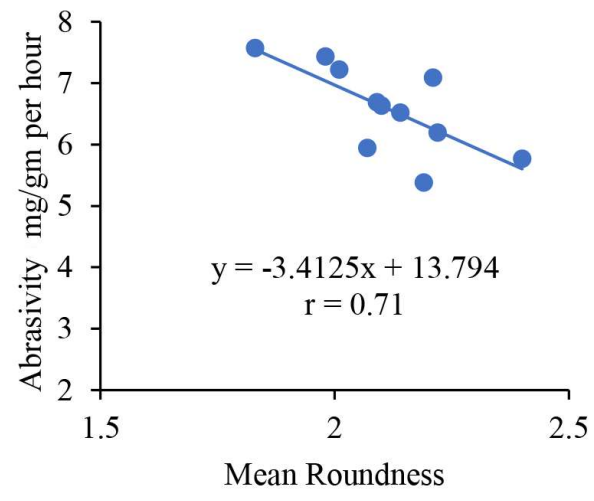

(a)

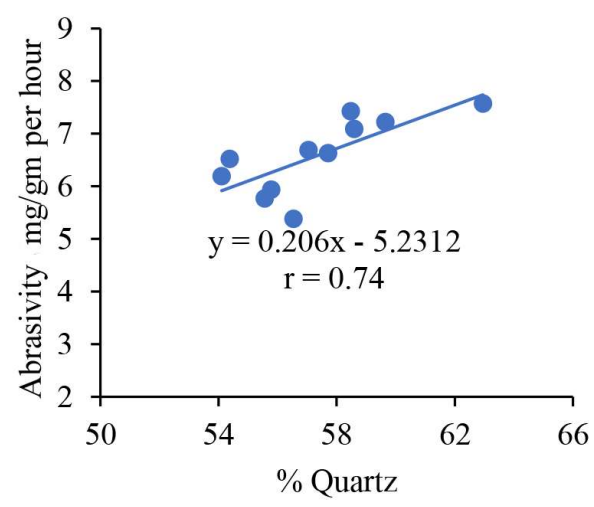

(c)

\section{DISCUSSIONS}

\section{Dependence of abrasivity upon texture and composition}

Both size fractions, 250 microns-pan and 500-250 microns, exhibit good correlations of abrasivity with mean roundness and \%quartz, moderate correlation with mean sphericity and good (in 250 microns-pan fraction) to poor (in 500-250-micron fraction) correlation with hardness index (Figs. 5 and 6). The correlation of abrasivity with mean roundness and sphericity is negative while that with \%quartz and hardness index is positive. When finer size fraction is considered, roundness and \%quartz and hardness index are good explanatory parameters. But for coarser size fraction, mean roundness is only the reasonable parameter to explain abrasivity. For all the fractions, roundness of particles is the reasonable variable that indicates that the abrasivity depends upon roundness of particles in sediment. Majority of the samples show subangular shapes, and it is quite serious that these samples are potential in abrading turbines. Poudel et al. (2012) studied the effect of sediment shape and size in hydraulic turbine material. They did tests and compared the Roshi Khola and the Indrawati River sediments and found that erosion was more severely recorded in the experiment of the samples having low sphericity.

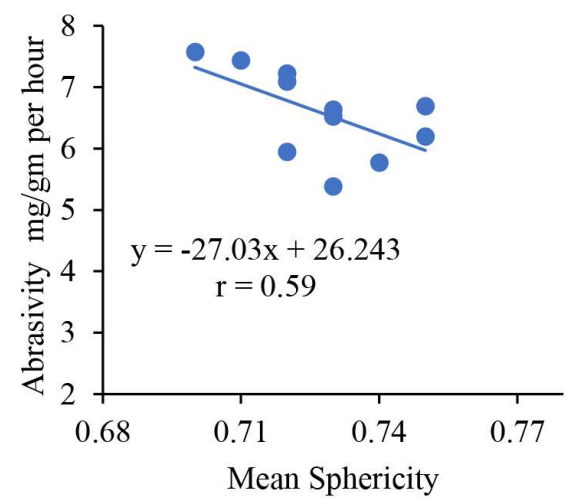

(b)

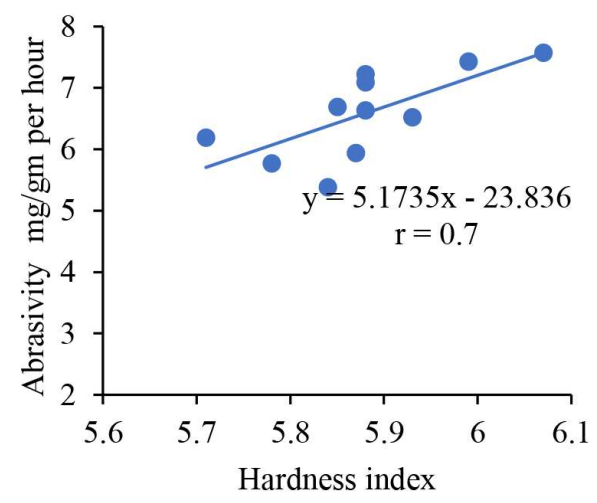

(d)

Fig. 5: Abrasivity of fraction size 250 microns-pan (a) Abrasivity, \% vs. Mean Roundness, (b) Abrasivity, \% vs. Mean Sphericity, (c) Abrasivity, \% vs. \% Quartz and (d) Abrasivity, \% vs. Hardness Index 


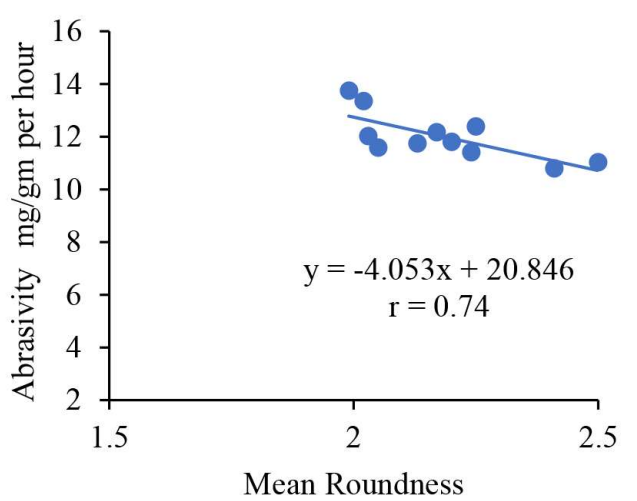

(a)

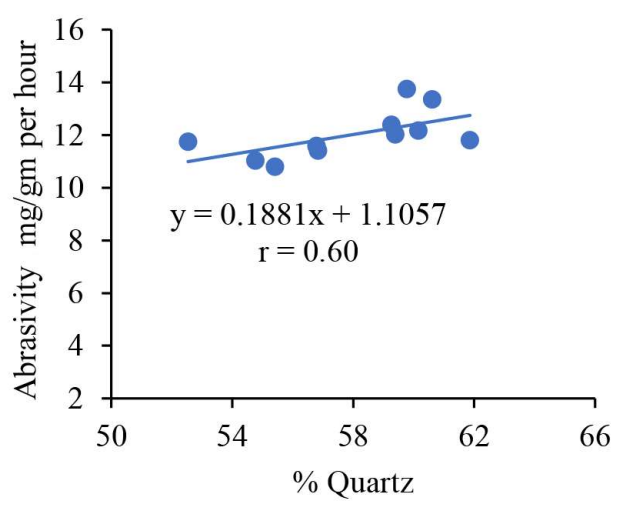

(c)

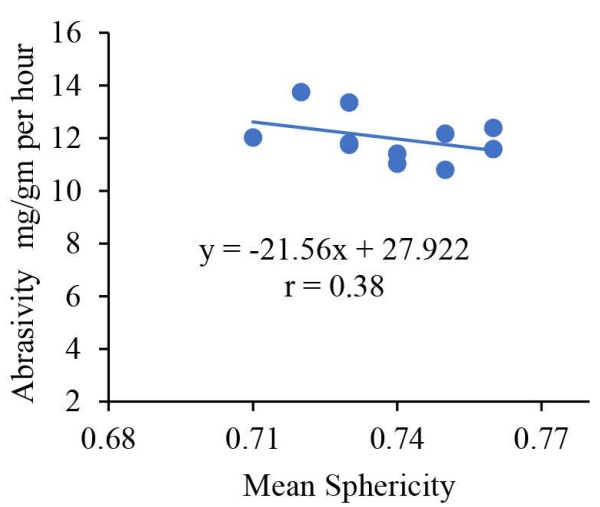

(b)

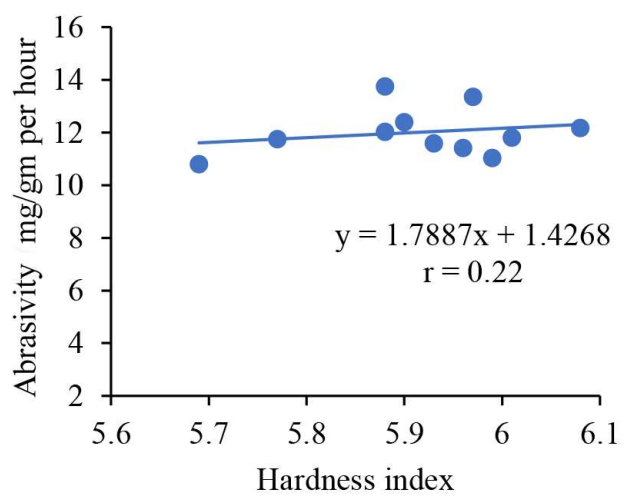

(d)

Fig. 6: Abrasivity of fraction size 500-250 microns (a) Abrasivity, \% vs. Mean Roundness, (b) Abrasivity, \% vs. Mean Sphericity, (c) Abrasivity, \% vs. \% Quartz, and (d) Abrasivity, \% vs. Hardness Index

Abrasion test on the specimen by sediment of the Budhi Gandaki and the confluence rivers were analysed in different ways. Truscott (1972) studied abrasive wear in hydraulic machinery and Thapa (2004) studied sediment erosion in hydroturbine component and concluded that erosion depended upon various factor, e.g., wear increased with grain size, shape, mineral composition, concentration and hardness etc.

\section{Analysis with respect to same size fraction at varying locations}

In 250 microns-pan size fraction, impact of the turbine is described in terms of abrasion rate. Comparing overall samples, in the Fig. 4 bar diagram shows the erosion impact is higher in upstream and is decreasing towards downstream of the river. Sample S5 has the highest abrasion rate $7.57 \mathrm{mg} / \mathrm{g}$ per hour. From this result it is depicted that the ample S5 at the Rampur Tar was more erosible than other samples because sample S5 was collected after the confluence of two local rivers. These river flows through weak geology having high crystalline, high grade metamorphic rock and brings sediments from a nearer source, which contributes more quartz mineral and angular grains. Sample S12 at Mugling has low abrasion rate $5.38 \mathrm{mg} / \mathrm{g}$ per hour. Trend line of 500-250-miron size fraction, shows more or less similar to the coarser size fraction, and abrasion rate decrease towards downstream of the river. Sample S5 at the Rampur Tar has the highest abrasion rate $13.75 \mathrm{mg} / \mathrm{g}$ per hour. Sample S14 at Ghumaune has low abrasion rate 10.80 $\mathrm{mg} / \mathrm{g}$ per hour. This is because sediment from downstream contains more sphered, less angular and low-grade soft minerals.

\section{Analysis with respect to varying fraction size but same location}

From the bar diagram Fig. 4, it can be easily understood that every sample in 500-250-micron size fraction sediment has the higher abrasion rate than 250 microns-pan size. From tabulated results 500-250-micron size sediments are almost twice more erosible than the smaller size fraction. Thus, it can be concluded that erosion depends upon size factor. 


\section{Abrasion rate compared with previous studies on turbine erosion}

The maximum abrasion rate of turbine specimen is 7.57 $\mathrm{mg} / \mathrm{g}$ per hour and minimum abrasion rate is $5.38 \mathrm{mg} / \mathrm{g}$ per hour when finer size fraction ( 250 mirons-pan) is considered as abrasive. But when 500-250-microns size fraction is considered in the experiment, the maximum abrasion rate of turbine specimen obtained is $13.75 \mathrm{mg} / \mathrm{g}$ per hour and minimum abrasion rate is $10.80 \mathrm{mg} / \mathrm{g}$ per hour in mild steel base material. Rajkarnikar (2013) used the test using the Sunkoshi River sand and found that the maximum abrasion rate was $21 \mathrm{mg} / \mathrm{g}$ per hour and minimum abrasion rate is $12.6 \mathrm{mg} / \mathrm{g}$ per hour. Bastola (2014) also studied sediment size 125 mirons-600 microns of the Marsyangdi River and found that abrasion rate was $16 \mathrm{mg} / \mathrm{g}$ per hour to $24 \mathrm{mg} / \mathrm{g}$ per hour. Both those hydropowers were facing severe abrasion of turbine requiring frequent maintainance (Thapa 2005). Mineral composition of the Marsyangdi River sediment is $61 \%-56 \%$ quartz and $10 \%$ feldspar. In both of the above-mentioned experiments authors used aluminium as the base material for the test specimens. If the test in the present study were done in aluminium base material then the abrasion rate would be maximum $27.5-21.6 \mathrm{mg} / \mathrm{g}$ per hour and minimum $15.14-10.76 \mathrm{mg} / \mathrm{g}$ per hour. These figures of abrasion rate are considered severe than the abrasive rate obtained in previous two studies, for the Sunkoshi and the Marsyangdi River sediment.

Design of settling basin of the Lower Marsyangdi Hydropower Project has $400 \mathrm{~m}$ long canal to trap $90 \%$ of particles greater than $0.06 \mathrm{~mm}$ grain size even though it is facing erosion of turbine components. The total sediment yield of the Marsyangdi River has 7,700 tonnes/sq. km/year (Shrestha, 2012). Settling basin of the Sunkoshi Hydropower has the efficiency to trap particles greater than $0.2 \mathrm{~mm}$ grain size and total sediment yield of Sunkoshi River has 5,400 tonnes/sq. $\mathrm{km} /$ year. But the total sediment yield in the Budhi Gandaki River is 3,390 tonnes/sq. km/year. In both hydropower project they have used High Velocity Oxygen Fuel (HVOF) coating to resist from erosion of the turbine but they do not show satisfactory results. If a hydropower plant is developed in the Budhi Gandaki river section having a similar design of settling basin, trapping efficiency and same turbine material, the HPP will have to face severe erosion than the erosion happened in the Lower Marsyangdi and the Sunkoshi Hydropower Projects. This study shows that the sediment of the Budi Gandaki-Trishuli River has high potential to abrasion of the turbine and its component.

\section{CONCLUSIONS}

1. Sediments of the Budhi Gandaki-Trishuli River are angular to sub angular with low sphericity.

2. Mineral composition of the Budhi Gandaki-Trishuli River is $54 \%-63 \%$ quartz, $8 \%-17 \%$ feldspar, $10 \%-20 \%$ mica, $5 \%-10 \%$ fine lithic fragments, $5 \%-12 \%$ coarse lithic fragments and others.

3. In both size fractions studied, the S5 Sample at the
Rampur Tar has the highest abrasivity but low abrasivity is observed in 250-pan size-fraction of S12 (Mugling) and in 500 -250-micron size fraction of S14 (Ghumaune).

4. Sediment size 500-250-microns is twice more erosive than 250 mirons-pan size sediment.

5. Abrasivity of the Budhi Gandaki River sediment is severe than the Sunkoshi and the Marsyangdi River sediment, and considering it, there should be taken due consideration of turbine material and construction design of settling basin to trap abrasive size-fractions.

6. Abrasivity of sediment is directly proportional to the size of grains, proportion of hard minerals whose hardness is 6 or greater and to the degree of mean roundness.

7. Abrasivity is better reflected by quartz content and mean roundness compared to hardness index and mean sphericity.

8. These findings will be useful for determining proper site of a power plant, suitable settling basins to trap sediment particles having higher erosion potentials and operational strategy to avoid sediment erosion.

\section{REFERENCES}

Bastola, A.K. and Neopane, H.P., 2014, Mineral Analysis and Erosion Potential of Sediment Samples from Nepalese Hydro Power Plant: A Case Study of Lower Marsyangdi Hydropower Plant. Journal of Machinery Manufacturing and Automation, v. 3, Issue no. 3, pp. 50-55.

Hayes, R.J., and Klugman, M.A., 1959, Feldspar staining methods. J. Sed. Petrol., v. 29, pp. 227-232. https://doi.org/10.1306/74D708E4-2B21-11D7$8648000102 \mathrm{C} 1865 \mathrm{D}$

Ingersoll, R.V. Bullard, T.F., Ford, R.L., Grimm, J.P., Pickle. J.D., and Sares, S.W., 1984, The defect of grain size on detrital modes: A test of the Gazzi-Dickinson Point counting method. Jour. Sediment. Petrol., v. 54, no. 1, pp.103-116. https://doi.org/10.1306/212F83B9-2B24-11D7$8648000102 \mathrm{C} 1865 \mathrm{D}$

Mann, S.B., 2000, High-energy particle impact wear resistance of hard coatings and their application in hydro-turbines. Wear v. 237, Issue no. 1, pp. 140-46. https://doi.org/10.1016/S0043-1648(99)00310-5

Parajuli, R., 2003, Theoretical hydroelectric capacity can be more Kantipur Daily (28/01/2003) (in Nepali).

Powers, M.C., 1953, A new roundness scale for sedimentary particles, J. Sed. Petrol., v. 3, pp. 117-136.

Padhy, M.K. and Saini, R.P., 2008, A review on silt erosion in hydro turbines. Renewable and Sustainable Energy Reviews, v. 12, no. 7, pp. 1974-1987. https://doi.org/10.1016/j.rser.2007.01.025

Poudel, L., 2012, Effect of Sediment Size in Hydraulic Turbine Material: A Case Study of Roshi Khola in Nepal. Nepal 
Journal of Science and Technology, v. 13, Issue no. 2, pp. 129-132. https://doi.org/10.3126/njst.v13i2.7725

Poudel, L., 2012, Sand Size impact on Hydraulic Turbine Material: A Case Study of Sunkoshi River. Nepal International Journal of Innovative Research in Technology and Science v. 1, no. 3, pp. 48-52.

Powers, M.C., 1953, A new roundness scale for sedimentary particles, J. Sed. Petrol., v. 23, pp. 117-136. https://doi.org/10.1306/D4269567-2B26-11D7$8648000102 \mathrm{C} 1865 \mathrm{D}$

Rajkarnikar, B., Neupane, H.P., and Thapa, B.S., 2013, Development of Rotating Disc Apparatus for test of sediment induced erosion in Francis runner blades. An International Journal on the Science and Technology of Friction, Lubrication, wear, v. 306, pp. 119-125. https://doi.org/10.1016/j.wear.2013.07.011

Rittenhouse, G., 1943, A visual method for estimating twodimensional sphericity. J. Sed. Petro., v. 13, no. 1, pp.
79-81.

Thapa, B., 2004a, Sand erosion in Francis turbine: A case study from Jhimruk power plant, Nepal, IAHR symposium on hydraulic machinery and systems, Stockholm, pp. 23-31.

Thapa, B., 2004b, Sand erosion in hydraulic machinery. PhD thesis, Norwegian University of Science and Technology, Faculty of Engineering Science and Technology, Department of Energy and Process Engineering, 226 p.

Thapa, B., Shrestha, R., Dhakal, P., and Thapa, B.S., 2005, Problems of Nepalese Hydropower Projects due to Suspended sediments. Journal of aquatic ecosystem Health and Management, v. 8, Issue no. 3, pp. 251-257. https://doi.org/10.1080/14634980500218241

Truscott, G.F., 1972, A literature survey on abrasive wear in hydraulic machinery. Wear, v. 20, pp. 29-50. https://doi.org/10.1016/0043-1648(72)90285-2 